\title{
A New Clinical Entity: IgG4-Related Disease (IgG4-RD) Discovered in the 21st Century
}

\author{
Hisanori Umehara \\ Key words: IgG4-related disease, Mikulicz's disease, autoimmune pancreatitis, pachymeningitis, IgG4-related \\ pulmonary disease
}

(Intern Med 51: 821-822, 2012)

(DOI: 10.2169/internalmedicine.51.7223)

IgG4-related disease (IgG4-RD) is a novel clinical disease entity characterized by an elevated serum IgG4 concentration and tumefaction or tissue infiltration by IgG4-positive plasma cells (1), and may be present among a certain proportion of patients formerly diagnosed as autoimmune pancreatitis $(2,3)$, Mikulicz's disease $(4,5)$, hypophysitis, Riedel thyroiditis, interstitial pneumonitis, interstitial nephritis, prostatitis, lymphadenopathy, retroperitoneal fibrosis, inflammatory aortic aneurysm and inflammatory pseudotumor. Although IgG4-RD forms a distinct, clinically independent disease category and is attracting strong attention as a new clinical entity, many questions and problems of IgG4-RD still remain to be elucidated, including its pathogenesis and the establishment of diagnostic criteria.

In consideration of this situation, two IgG4-RD study groups have been organized by the Ministry of Health, Labor and Welfare (MHLW) Japan. One of these groups, chaired by Professor Umehara of Kanazawa Medical University (Umehara team, 66 members), is seeking to establish the diagnostic criteria for IgG4-related multi-organ lymphoproliferative syndrome (IgG4-MOLPS), whereas the second group, chaired by Professor Okazaki of Kasai Medical University (Okazaki team, 55 members), is seeking to understand the etiology and pathogenesis of IgG4-related systemic disease. These two groups consist of doctors and researchers in various fields, including rheumatology, hematology, gastroenterology, nephrology, pulmonology, ophthalmology, odontology, pathology, statistics, basic and molecular immunology from all over Japan. In addition, collaborations of the two study groups (all Japan IgG4 team) have gathered the detailed analyses of the clinical symptoms, laboratory results and biopsy specimens of patients with IgG 4-RD, resulting in the establishment of general concepts for IgG4-RD (1). A consensus for IgG4-RD is that it can occur in various organs, including the central nervous system, sali- vary glands, thyroid gland, lungs, pancreas, biliary duct, liver, gastrointestinal tract, kidneys, prostate gland, retroperitoneum, and lymph nodes, but that clinical symptoms are dependent upon the lesion location. IgG4-RD mainly affects middle-aged to elderly men. Its clinical symptoms are relatively mild, and the condition usually comes to clinical attention as a result of organ swelling or damage. Many patients with IgG4-RD are treated effectively by steroid therapy. Although the infiltration of IgG4-positive cells and increased serum concentrations of IgG4 are characteristic of IgG4RD, the severity of fibrosis is dependent upon the individual organs involved.

In this issue of Internal Medicine, Yamashita et al. reported a rare case of IgG4-RD in combination with Mikulicz's disease, hypertrophic pachymeningitis and pulmonary involvement (6). In addition, all of such involvements responded well to glucocorticoid treatment. Since IgG 4-related pachymeningitis is very rare itself and it is difficult to obtain tissue samples, their case report is suggestive of a diagnosis IgG4-RD with such conditions.

Although there is increased interest in IgG4-RD, awareness of it remains low and the diagnostic criteria have not yet been published. Very recently, the all Japan IgG4 team has established comprehensive diagnostic criteria for IgG4 RD consisting of only three items (7): 1) organ enlargement, mass or nodular lesions, or organ dysfunction, 2) a serum IgG4 concentration $>135 \mathrm{mg} / \mathrm{dL}$, and 3) histopathological findings of $>10 \mathrm{IgG} 4+$ cells/HPF and an $\mathrm{IgG} 4+/ \mathrm{IgG}+$ cell ratio $>40 \%$ (Table 1). The comprehensive diagnostic criteria for IgG4-RD are practically useful for general physicians and non-specialists. Although it is difficult to obtain tissue biopsy samples from some organs, including the pancreas, retroperitoneum and brain, patients who can not be diagnosed by the comprehensive diagnostic criteria could be diagnosed by organ-specific criteria, such as IgG4-related 
Table 1. Comprehensive Clinical Diagnostic Criteria for IgG4-RD (7)

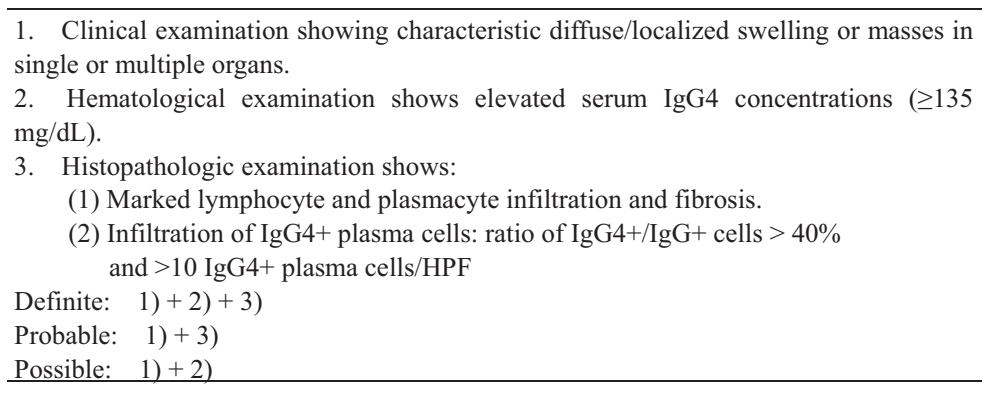

AIP (8), IgG4-related Mikulicz's disease (9) and IgG4related kidney disease (10), indicating the complementarity of comprehensive diagnostic criteria and organ-specific criteria for the diagnosis of IgG4-RD.

\section{The author states that he has no Conflict of Interest (COI).}

\section{References}

1. Umehara H, Okazaki K, Masaki $\mathrm{Y}$, et al. A novel clinical entity, IgG4-related disease (IgG4RD): general concept and details. Mod Rheumatol 2011 [Epub ahead of print], PMID: 21881964.

2. Hamano H, Kawa S, Horiuchi A, et al. High serum IgG4 concentrations in patients with sclerosing pancreatitis. N Engl J Med 344: 732-738, 2001.

3. Kamisawa T, Funata N, Hayashi Y, et al. A new clinicopathological entity of IgG4-related autoimmune disease. J Gastroenterol $\mathbf{3 8}$ 982-984, 2003.

4. Yamamoto M, Ohara M, Suzuki C, et al. Elevated IgG4 concentrations in serum of patients with Mikulicz's disease. Scand J Rhumatol 33: 432-433, 2004.
5. Masaki Y, Dong L, Kurose N, et al. Proposal for a new clinical entity, IgG4-positive multi-organ lymphoproliferative syndrome: Analysis of 64 cases of IgG4-related disorders. Ann Rheum Dis 63: 1310-1315, 2009.

6. Yamashita H, Takahashi Y, Ishiura H, et al. Hypertrophic pachymeningitis and tracheobronchial 1 stenosis in IgG4-related disease: case presentation and literature review. Intern Med 51: 935-941, 2012.

7. Umehara H, Okazaki K, Masaki Y, et al. Comprehensive diagnostic criteria for IgG4-related disease (IgG4-RD). 2011. Mod. Rheumatol. [Epub ahead of print] PMID: 22218969.

8. Okazaki K, Kawa S, Kamisawa T, et al. Clinical diagnostic criteria of autoimmune pancreatitis: revised proposal. J Gastroenterol 41: 626-631, 2006

9. Masaki Y, Sugai S, Umehara H. IgG4-related diseases including Mikulicz's disease and sclerosing pancreatitis: diagnostic insights. J Rheum 37: 2010.

10. Kawano M, Saeki T, Nakashima H, et al. Proposal for diagnostic criteria for IgG4-related kidney disease. Clin Exp Nephrol 15: 615-626, 2011

(C) 2012 The Japanese Society of Internal Medicine http://www.naika.or.jp/imindex.html 